\title{
Dispersion Inversion of Guided P Waves in a Waveguide of Arbitrary Geometry Jing $\mathrm{Li}^{* \dagger}$, Sherif Hanafy*, and Gerard T. Schuster* \\ * King Abdullah University of Science and Technology, Thuwal, Saudi Arabia, 23955-6900. \\ $\dagger$ College of Geo-Exploration Science and Technology, Jilin University, Changchun 130026.
}

\section{SUMMARY}

We present the theory for wave equation inversion of dispersion curves obtained from traces containing guided $\mathrm{P}$ waves. The misfit function is the sum of the squared differences between the wavenumbers along the predicted and observed dispersion curves, and the inverted result is a high-resolution estimate of the near-surface P-velocity model. This procedure, denoted as the wave equation dispersion inversion of guided $\mathrm{P}$ waves (WDG), is valid for near-surface waveguides with irregular layers. It is less prone to the cycle skipping problems of full waveform inversion (FWI) and can sometimes provide velocity models with higher resolution than wave-equation traveltime tomography (WT). The synthetic and field data examples demonstrate that WDG for guided $\mathrm{P}$ waves can accurately reconstruct the $\mathrm{P}$-wave velocity distribution in laterally heterogeneous media.

\section{INTRODUCTION}

A waveguide is a structure that guides waves, such as electromagnetic waves or sound, with minimal loss of energy by restricting the wave propagation to one or two dimensions. For a source on the free surface of a quasi-layered earth, the generated guided waves mainly propagate along the interfaces. The guided waves are mainly post-critical reflections and refractions because the deepest layer has the fastest velocity (Roth et al., 1998). The main characteristics of guided waves are that the propagating waves are dispersive and their geometrical spreading behaves as $O\left(r^{-1 / 2}\right)$, where $r$ is the horizontal distance between the point source and receiver on the surface.

As an example, Figure 1a shows the recordings of the guided waves (GW) that arrive just after the first arrival. We denote such events as "railroad tracks", because their traveltime curves are almost parallel to those of the P-wave refraction arrivals. These arrivals include P-wave reflections and refractions in the topmost system of layers. Such waves disperse as they are trapped in the near-surface low-velocity layer, and the corresponding dispersion curves are shown in Figure $1 b$.

The much slower arrivals in Figure 1a are the surface waves denoted as SW and propagate according to the S-velocity of the associated layers. These surface waves are also known as guided waves because they are mainly post-critical P-SV reflections trapped in the near-surface layers. Their corresponding dispersion curves, denoted as SW in Figure 1b, show that they are much slower than the guided $P$ waves denoted as GW.

We now present the WDG (in our case, denoted as wave equation dispersion inversion of guided waves (WDG)) method eliminates the high-frequency assumption of ray-based tomogra-
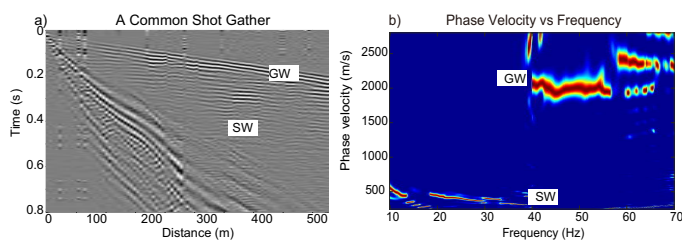

Figure 1: a) Common shot gather recorded over the Qademah fault system near KAUST. The "railroad" tracks of guidedwave arrivals $(\mathrm{GW})$ that trail just behind the head-wave arrivals indicate resonance in the topmost layers. The surface-wave arrivals are labeled as SW. b) The spectrum of the shot gather reveals the dispersion curves for the guided waves $(\mathrm{GW})$ and the surface waves (SW).

phy methods, removes the layered media approximation of standard inversion of dispersion curves (Boiero et al., 2013a; Campbell et al., 2017) and mitigates FWI's tendency of getting stuck in a local minimum (Mora, 1987; Virieux and Operto, 2009). As we wiil show, WDG inverts for the P-velocity distribution from guided P-wave dispersion curves. These types of guided waves can exist for a model that consists of a waveguide with irregular layers just below the free surface. The thickness of these layers should be larger than the characteristic wavelength of the source so that waves can propagate at frequencies above the cut-off frequency (Grant and West, 1965).

WDG is similar to the wave equation dispersion inversion (WD) method of Li and Schuster (2016). The WD method is typically used to invert for the S-velocity associated with surfacewave dispersion curves and a layered medium with irregular interfaces. Now, we invert for the P-velocity model from the guided $\mathrm{P}$ waves in the shallow layers. No Born, highfrequency or $1 \mathrm{D}$ approximations are required, and it is less prone to getting stuck in local minima compared to FWI. As noted by Li et al. (2017a), the WD procedure is more robust than FWI because it replaces complicated guides-waves arrivals with simple dispersion curves in the wavenumber $k_{x}-\omega$ or phase-velocity $C(\omega)-\omega$ domains. These dispersion curves are considered to be skeletonized data (Luo and Schuster, 1991b,a).

\section{THEORY OF SKELETONIZED INVERSION OF GUIDED WAVES}

The WDG gradient formulas are computed by finite-difference solutions to the elastic wave equation, but in practice we often use solutions to the elastic-wave equation.

The dispersion misfit function $\varepsilon$ is defined as the sum of squared 


\section{Guided waves WD}

dispersion curves residuals:

$$
\varepsilon=\frac{1}{2} \sum_{\omega} \overbrace{\left(\kappa(\omega)-\kappa(\omega)^{o b s}\right.}^{\text {residual }=\Delta \kappa(\omega)},
$$

so that the optimal P-slowness model $s(\mathbf{x})$ is obtained from the iterative steepest descent solution:

$$
s(\mathbf{x})^{(k+1)}=s(\mathbf{x})^{(k)}-\alpha \sum_{\omega} \Delta \kappa(\omega) \frac{\partial \kappa(\omega)}{\partial s(\mathbf{x})},
$$

where $s(\mathbf{x})=1 / c(\mathbf{x}), \alpha$ is the step length and the superscript $(k)$ denotes the $k^{\text {th }}$ iteration. For pedagogical simplicity, we assumed a single shot gather, but the misfit function includes an additional summation over different shot gathers if more than one shot gather is used. In practice we use a preconditioned conjugate gradient optimization method. A penalty function can be incorporated into the misfit function to penalize variations of the velocity model faster than the smallest wavelength in the data.

\section{Resolution and Higher-Order Modes}

Higher-order guided waves can be more sensitive to changes in the medium parameters than the lower-order modes. This claim can be demonstrated in the case of an acoustic slab with a free surface on the top and a rigid boundary at the bottom, and the slab thickness is $h$. In this case, Grant and West (1965) show that the horizontal phase slowness $1 / C(\omega)$ of a specified mode in the frequency domain is given by

$$
S(\omega)=\frac{1}{C(\omega)}=\left[\frac{1}{c^{2}}-\frac{(n+1 / 2)^{2} \pi^{2}}{\omega^{2} h^{2}}\right]^{1 / 2},
$$

where $n$ is the mode number, $C(\omega)$ is the phase velocity of a specified mode, $c$ is the $\mathrm{P}$-wave velocity, and $\omega$ is the angular frequency. Differentiating the phase-slowness in equation 3 with respect to the slab thickness $h$ gives:

$$
\frac{\partial S(\omega)}{\partial h}=\frac{(n+1 / 2)^{2} \pi^{2}}{S(\omega) \omega^{2} h^{3}} .
$$

This equation says that the apparent slowness becomes more sensitive to changes in the thickness $h$ for increasing mode numbers. As an example, Figure 2 displays the dispersion curves for an acoustic slab, where the slope of the $n=1$ curve at the frequency $f_{o}$ is steeper than the slope for the $n=0$ curve. The large value of the slope at $f_{o}$ for $n=1$ indicates a strong sensitivity of the data, i.e. changes in $S(\omega)$, to changes in the slab thickness. Thus, the WDG method has the capability for high resolution imaging of the slab thickness. This is not too surprising because the steep part of the dispersion curves corresponds to nearly vertically incident waves, which generate resonant reflections that can provide superresolution imaging Schuster and Huang (2014).

The formula that relates depth resolution $\delta h$ to mode number $n$ can be obtained by defining the phase $\phi(x, t, \omega)$ of the traveling guided wave as

$$
\phi(x, t, \omega)=\omega[x S(\omega)-t],
$$

Dispersion Curves for Acoustic Slab

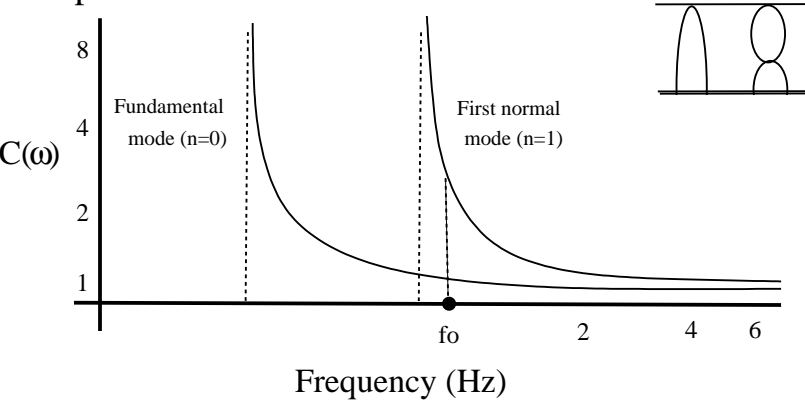

Figure 2: Dispersion curves associated with an acoustic slab of thickness $h$ with a free surface on the top and a rigid boundary condition on the bottom. Illustration adapted from Grant and West (1965).
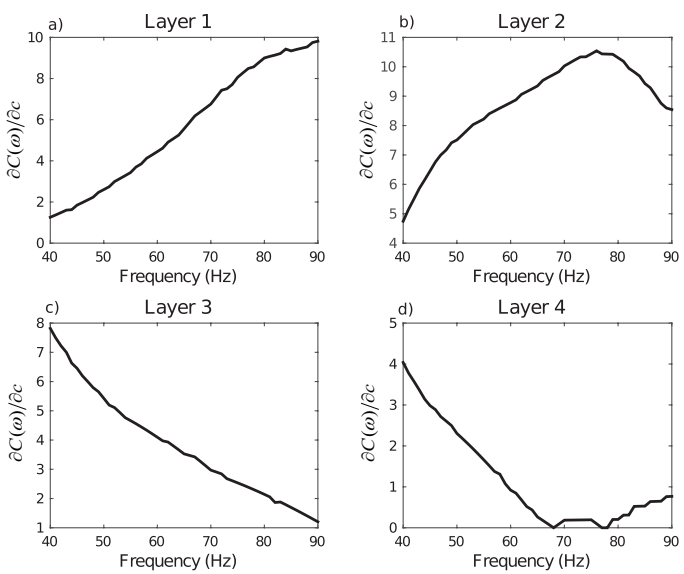

Figure 3: Normalized phase velocity derivatives with respect to P-wave velocity variations $\partial C(\omega) / \partial c$ in the a) first, b) second, c) third, and d) fourth layers.

perturbing $\phi$ in equation 5 by $\delta h$, inserting equation 13, and after some rearrangement we get

$$
\delta h=\frac{\delta \phi \omega h^{3}}{x} \frac{\left[\frac{1}{v_{p}^{2}}-\frac{(n+1 / 2)^{2} \pi^{2}}{\omega^{2} h^{2}}\right]^{1 / 2}}{(n+1 / 2)^{2} \pi^{2}},
$$

where $\delta \phi$ is the phase perturbation due to a change in layer thickness $\delta h$. If $\delta \phi$ is set to a detectable level in the data, then we conclude that $\delta h$ gets smaller as the mode order $n$ becomes larger. This suggests that small changes in $\delta h$ can be detected with the detectable change in the phase $\delta \phi$. Hence, superresolution estimation of the slab thickness might be possible by inverting higher-order modes in the dispersion curves.

\section{NUMERICAL TESTS}

\section{Sensitivity Analysis}

Firstly, we use a four-layered model to test the sensitivity of the 

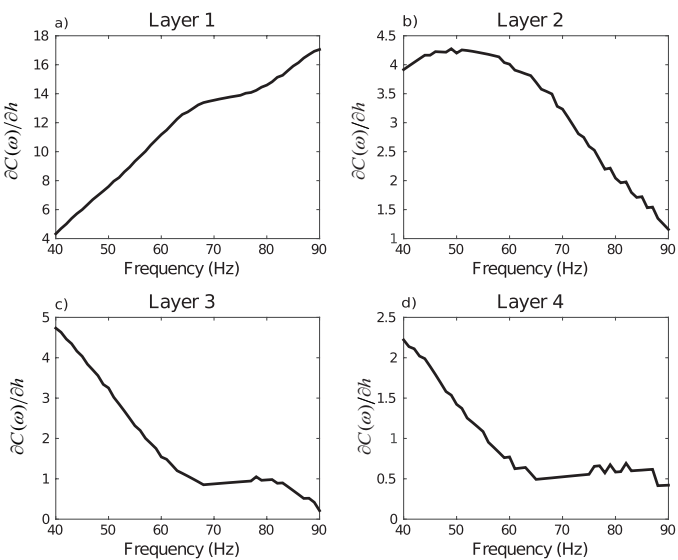

Figure 4: Normalized phase velocity derivatives with respect to thickness variations $\partial C(\omega) / \partial h$ in the a) first, b) second, c) third, and d) fourth layers.

phase velocity $C(\omega)$ with respect to changes of the P-wave velocity $\partial C(\omega) / \partial c(x)$ and the layer thickness $\partial C(\omega) / \partial h$, where $c(\mathbf{x})$ and $h$ represent the P-wave velocity and layer thickness, respectively. The result plotted in Figure 3 shows that the sensitivity of the phase velocity with respect to changes in the $\mathrm{P}$-velocity is frequency-dependent, where the phase velocity variations $\partial C / \partial c$ in the shallower (deeper) layers are more sensitive to higher (lower) frequencies. This indicates that lower frequencies are needed to image deeper structures in the near surface. This is also valid for estimating layer thicknesses as shown in Figure 4, where the low-frequency phase-velocity derivatives $\partial C / \partial h$ are most sensitive to the thickness variations in the deeper layers (Boiero et al., 2013b; Campbell et al., 2017).

\section{Complex Three-Layered Model}

Data are now generated for the complex three-layer velocity model in Figure 5a to assess the resolution capabilities of the WDG method. The first layer interface is at the depth of $2 \mathrm{~m}$ with the P-wave velocities $c_{1}=900 \mathrm{~m} / \mathrm{s}$ and $c_{2}=1100 \mathrm{~m} / \mathrm{s}$, respectively. The guided waves have very weak amplitudes due to the subtle velocity contrasts.

The second interface is at the depth of $8 \mathrm{~m}$ and the P-wave velocity of the third layer is $1800 \mathrm{~m} / \mathrm{s}$. Here, the guided waves are trapped above the second interface, and have strong amplitudes. A Ricker wavelet with a $30-\mathrm{Hz}$ peak frequency is used as the source wavelet for generating this data, and has the dominant wavelength of $37 \mathrm{~m}$ in the second layer. We still use the gradient velocity model as the initial model and the inverted model is shown in Figure 5b, where the white dashed lines depict the velocity interfaces. The predicted dispersion curves shown in Figure 6a (black solid lines) agree with the observed values shown in Figure 6a (black dashed lines), and the normalized misfit function plotted in Figure $6 \mathrm{~b}$ shows a rapid convergence. However, the WDG method can not reconstruct the topmost thin layer which is due to the weak velocity contrast that is not able to generate the guided waves with strong amplitudes.
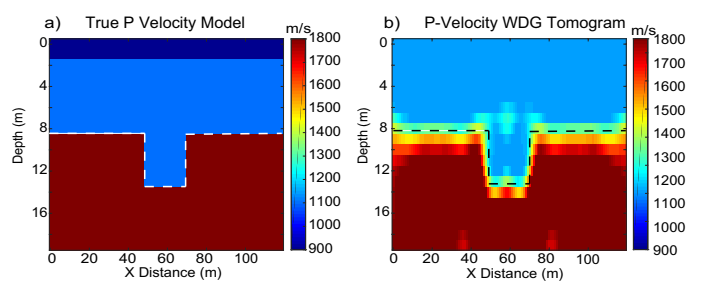

Figure 5: a) True P-velocity model and b) P-velocity tomogram after 25 iterations of the WDG method.
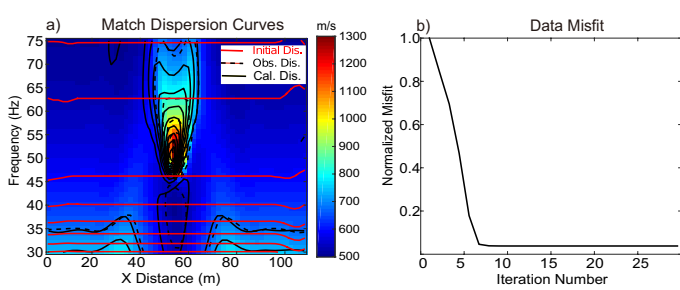

Figure 6: Plots of a) dispersion curves for all of the predicted and observed shot gathers and $b$ ) normalized misfit function vs iteration number.

\section{Qademah Field Data}

The WDG method is applied to seismic data recorded over a hidden low-velocity zone (LVZ) near the King Abdullah Economic City (KAEC) in Saudi Arabia. This low-velocity zone is denoted as the Qademah fault and strikes in a north-south direction. The direct evidence for the fault is not seen at this location but it is inferred from the geophysical data as a LVZ (Hanafy et al., 2015; Li and Hanafy, 2016). The shot spacing is $5 \mathrm{~m}$, the $\mathrm{y}$-component receiver spacing is $2.5 \mathrm{~m}$, and there are 240 traces per shot gather to give a total of 120 shot gathers. A $90 \mathrm{~kg}$ weight drop is used as the seismic source, with 10 to 15 stacks at each shot location. Inverting the Love waves gives a low-S-velocity zone (LVZ) above $20 \mathrm{~m}$ in the middle of site 2 (Li et al., 2017b).

The guided P-wave dispersion curves are inverted to get the $\mathrm{P}$-velocity tomogram. Figure $7 \mathrm{a}$ shows the $\mathrm{P}$-wave tomogram computed by first-arrival wave equation tomography (WT) with the nearly vertical dashed lines interpreted from the zero-offset common offset gathers (COGs) in Figure $7 \mathrm{c}$. Figure $7 \mathrm{~b}$ shows the $\mathrm{P}$-wave velocity tomogram inverted from the guided-wave dispersion curves. This interpretation is consistent with the red dashed lines seen in the COGs (Figure 7c). Here, a LVZ is suggested by a rapid lateral change in the P-velocity values.

The WT tomogram in Figure 7a indicates a blocky high-velocity structure at the offset of about $100 \mathrm{~m}$, and it also shows a mostly horizontal interface with offset $150 m<x<600 m$ and depth of $z \approx 10 \mathrm{~m}$. In contrast, the WDG tomogram in Figure $7 \mathrm{~b}$ shows a more rounded high-velocity zone. In addition, the WDG tomogram shows a low velocity (blue colors at $0<z<10 \mathrm{~m}$ ) zone that becomes deeper from the offset $x=600 \mathrm{~m}$ to the area around $x=200 \mathrm{~m}$. This dipping lowvelocity feature appears to be more consistent with the zerooffset COG in Figure 7c where there is an increasing delay 


\section{Guided waves WD}
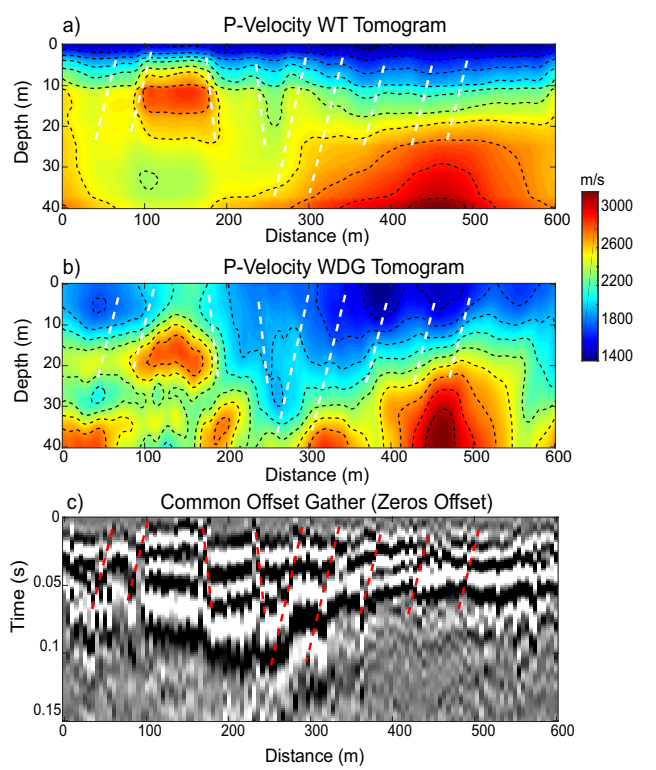

Figure 7: a) P-velocity tomogram by multiscale wave equation traveltime inversion of first arrivals, b) P-velocity tomogram obtained by inverting the guided $\mathrm{P}$-wave the dispersion curves of the Qademah data, and c) COGs at the source-receiver offset of $0 \mathrm{~m}$. Dashed lines indicate abrupt discontinuities in the reflectivity interface, which might indicate the presence of cracks, faults or LVZs in the subsurface.

in the reflection events from right to left. The high-velocity zone (HVZ) in both tomograms is in agreement with the zerooffset common offset gather (ZOG) in Figure 7c, which indicates faults, cracks or LVZs adjacent to the high-velocity zone (HVZ). The ZOG indicates a sharply defined velocity contrast at the HVZ, which is more consistent with the WT tomogram. However, the ZOG does not clearly indicate a sharp horizontal interface at $z=10 m$ and $150 m<x<600 m$.

As a final sanity test, Figure 8 compares the predicted COGs computed from the WDG and WT tomograms to the recorded COG. The COG predicted from the WT tomogram simulates the smoothly varying events in the recorded COG, but is not able to capture the sudden jumps in the recorded events (Figure $8 \mathrm{c}$ ). However, the WDG COG profile (offset=50 $\mathrm{m}$ ) in Figure $8 \mathrm{~b}$ is more consistent with such jumps in the recorded COG in Figure 8a.

\section{SUMMARY}

We present the theory for wave equation inversion of guided $\mathrm{P}$-wave dispersion curves (WDG), where the dispersion misfit function is the sum of the squared wavenumber differences between the predicted and observed dispersion curves. The $\mathrm{P}$-wave velocity model is updated by migrating the weighted data, where the weight is proportional to the dispersion residual. Our synthetic and field data examples suggest that WDG is effective for inverting for 2D P-velocity models where the dispersion curves can be readily identified. The comparison
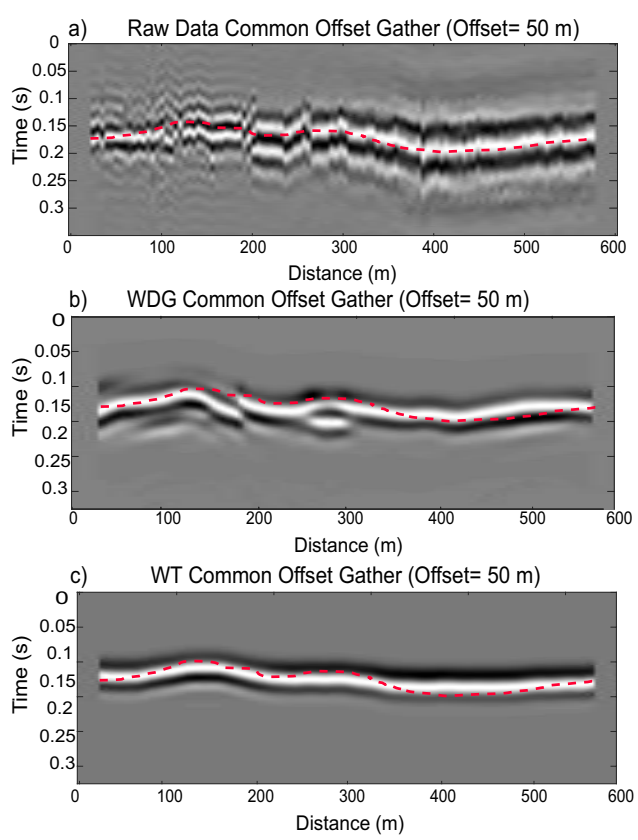

Figure 8: Comparison of a) the recorded COG, b) and c) the synthetic COGs computed from the WDG and WT tomograms, respectively. In this example the source-receiver offset is $50 \mathrm{~m}$ and the dashed red line is picked from the maximum amplitudes in the COG shown in a).

between the WT and WDG tomograms suggests that the COG simulated from the WDG tomogram is more consistent with the recorded data than the WT tomogram. Future research should explore the possibility of getting better resolution by inverting the higher-order modes in the dispersion curves. There are several challenges with the WDG method.

1. WDG cannot invert for the P-velocity unless there are significant velocity contrasts in the low-velocity zone.

2. WDG requires a wide-frequency band so that the thickness of the thinnest layer is larger than about $1 / 4$ of the shortest wavelength.

3. If the near-surface model is too complex, then coherent dispersion curves might not be identifiable. Further tests are needed to assess the robustness of this method for more complex models.

4. The final dispersion tomogram is sensitive to the accuracy of the picked dispersion curve and the offset range of traces used for calculating the Radon transform.

\section{ACKNOWLEDGEMENTS}

We thank the financial support from the sponsors of the Consortium of Subsurface Imaging and Fluid Modeling (CSIM) and the supercomputing center at KAUST for computational resources. We also thank the China Postdoctoral Science Foundation 2106T902503 and 2015M571366. 


\section{REFERENCES}

Boiero, D., C. Strobbia, L. Velasco, and P. Vermeer, 2013a, Guided waves-Inversion and attenuation: 75th Annual International Conference and Exhibition, EAGE, Extended Abstracts, https://doi.org/10.3997/2214-4609.20130008.

Boiero, D., E. Wiarda, and P. Vermeer, 2013b, Surface- and guided-wave inversion for near-surface modeling in land and shallow marine seismic data: The Leading Edge, 32, 638-646, https://doi.org/10.1190/tle32060638.1.

Campbell, K., R. Askari, W. Pennington, and A. Javahery, 2017, Estimation of the P-wave velocity of the near surface using dispersion analysis of the guided P-wave: 79th Annual International Conference and Exhibition, EAGE, Extended Abstracts, https://doi.org/10.3997/2214-4609.201701180.

Grant, F. S., and G. F. West, 1965, Interpretation theory in applied geophysics: McGraw-Hill.

Hanafy, S. M., A. AlTheyab, and G. T. Schuster, 2015, Controlled noise seismology: 85th Annual International Meeting, SEG, Expanded Abstracts, 5102-5106, https://doi.org/10.1190/segam2015-5906063.1.

Li, J., Z. Feng, and G. T. Schuster, 2017a, Wave-equation dispersion inversion: Geophysical Journal International, 208, 1567-1578, https://doi.org/10 $1093 /$ gji/ggw465.

Li, J., and S. Hanafy, 2016, Skeletonized inversion of surface wave: Active source versus controlled noise comparison: Interpretation, 4, no. 3, SH11-SH19, https://doi.org/10.1190/INT-2015-0174.1.

Li, J., and G. Schuster, 2016, Skeletonized wave equation of surface wave dispersion inversion: 86th Annual International Meeting, SEG, Expanded Abstracts, 3630-3635, https://doi.org/10.1190/segam2016-13770057.1.

Li, J., H. Sherif, and G. T. Schuster, 2017b, Wave-equation dispersion inversion of Love waves: Geophysics (in review)

Luo, Y., and G. T. Schuster, 1991a, Wave equation inversion of skeletalized geophysical data: Geophysical Journal International, 105, 289-294, https://doi.org/10.1111/j.1365-246X.1991.tb06713.x.

Luo, Y., and G. T. Schuster, 1991b, Wave-equation traveltime inversion: Geophysics, 56, 645-653, https://doi.org/10.1190/1.1443081.

Mora, P., 1987, Nonlinear two-dimensional elastic inversion of multioffset seismic data: Geophysics, 52, 1211-1228, https://doi.org/10.1190/1 .1442384 .

Roth, M., K. Holliger, and A. Green, 1998, Guided waves in near-surface seismic surveys: Geophysical Research Letters, 25, 1071-1074, https://doi org/10.1029/98GL00549.

Schuster, G. T., and Y. Huang, 2014, Far-field superresolution by imaging of resonance scattering: Geophysical Journal International, 199, 1943-1949, https://doi.org/10.1093/gii/ggu350.

Virieux, J., and S. Operto, 2009, An overview of full-waveform inversion in exploration geophysics: Geophysics, 74, no. 6, WCC1-WCC26, https:// doi.org/10.1190/1.3238367. 